\title{
Determination of cup-to-disc ratio of optical nerve head for diagnosis of glaucoma on stereo retinal fundus image pairs
}

Chisako Muramatsu, Toshiaki Nakagawa, Akira Sawada, Yuji Hatanaka, Takeshi Hara, et al.

Chisako Muramatsu, Toshiaki Nakagawa, Akira Sawada, Yuji Hatanaka, Takeshi Hara, Tetsuya Yamamoto, Hiroshi Fujita, "Determination of cup-todisc ratio of optical nerve head for diagnosis of glaucoma on stereo retinal fundus image pairs," Proc. SPIE 7260, Medical Imaging 2009: ComputerAided Diagnosis, 72603L (3 March 2009); doi: 10.1117/12.811461

Event: SPIE Medical Imaging, 2009, Lake Buena Vista (Orlando Area), Florida, United States 


\title{
Determination of cup and disc ratio of optical nerve head for diagnosis of glaucoma on stereo retinal fundus image pairs
}

\author{
Chisako Muramatsu*a, Toshiaki Nakagawa ${ }^{\mathrm{b}}$, Akira Sawada ${ }^{\mathrm{c}}$, \\ Yuji Hatanaka ${ }^{\mathrm{d}}$, Takeshi Hara ${ }^{\mathrm{a}}$, Tetsuya Yamamoto ${ }^{\mathrm{c}}$, Hiroshi Fujita ${ }^{\mathrm{a}}$ \\ ${ }^{a}$ Dept. of Intelligent Image Information, Graduate School of Medicine, Gifu University, \\ 1-1 Yanagido, Gifu, Japan 501-1194; \\ ${ }^{b}$ Kowa Company, Ltd., 3-3-1 Chofugaoka, Chofu, Tokyo, Japan 182-0021; \\ ${ }^{\mathrm{c} D e p t}$. of Ophthalmology, Graduate School of Medicine, Gifu University, \\ 1-1 Yanagido, Gifu, Japan 501-1194; \\ ${ }^{\mathrm{d}}$ School of Engineering, The University of Shiga Prefecture, \\ 2500 Hassaka-cho, Hikone, Shiga, Japan 522-0057
}

\begin{abstract}
A large cup-to-disc (C/D) ratio, which is the ratio of the diameter of the depression (cup) to that of the optical nerve head $(\mathrm{ONH}$, disc), can be one of the important signs for diagnosis of glaucoma. Eighty eyes, including 25 eyes with the signs of glaucoma, were imaged by a stereo retinal fundus camera. An ophthalmologist provided the outlines of cup and disc on a regular monitor and on the stereo display. The depth image of the ONH was created by determining the corresponding pixels in a pair of images based on the correlation coefficient in localized regions. The areas of the disc and cup were determined by use of the red component in one of the color images and by use of the depth image, respectively. The $\mathrm{C} / \mathrm{D}$ ratio was determined based on the largest vertical lengths in the cup and disc areas, which was then compared with that by the ophthalmologist. The disc areas determined by the computerized method agreed relatively well with those determined by the ophthalmologist, whereas the agreement for the cup areas was somewhat lower. When C/D ratios were employed for distinction between the glaucomatous and non-glaucomatous eyes, the area under the receiver operating characteristic curve (AUC) was 0.83 . The computerized analysis of ONH can be useful for diagnosis of glaucoma.
\end{abstract}

Keywords: Glaucoma, Stereo retinal fundus images, computerized analysis, cup-to-disc ratio

\section{INTRODUCTION}

Eye examination using retinal fundus images is frequently employed for evaluation of ocular diseases. The fundus images is also taken in the health checkups for its advantages in low cost, non-invasiveness, and simplicity, and can be useful for early detection of hypertensive retinopathy, diabetic retinopathy, and glaucoma. However, the recent increase in the number of examinations resulted in an increased burden on ophthalmologists. Moreover, in the screening exams, it is seldom that the diagnosis is made by specialists. The presentation of the results of computerized analysis can be useful in these situations. It has been our interest in developing the computer-aided diagnosis (CAD) system for fundus examinations [1-3], which includes the detection of optic nerve fiber layer defects (NFLD), hemorrhages, microaneurysms, and stenosis of blood vessels.

Glaucoma is one of the major causes of permanent blindness, especially in the developed countries [4], and its incident rate is expected to increase [5]. The study [6] reported that the probability of glaucoma patients' becoming blind in at least one eye was estimated to be $27 \%$. However, because of its gradual progression, many patients with glaucoma are unrecognized until the disease gets to an advanced stage. It is important, therefore, to diagnose the glaucoma early when the treatment can be effective.

*chisa@fjt.info.gifu-u.ac.jp; phone +81-58-230-6519; fax +81-58-230-6514; www.fjt.info.gifu-u.ac.jp

Medical Imaging 2009: Computer-Aided Diagnosis, edited by Nico Karssemeijer, Maryellen L. Giger Proc. of SPIE Vol. 7260, 72603L - (C) 2009 SPIE · CCC code: 1605-7422/09/\$18 - doi: 10.1117/12.811461 
The major risk factor for most glaucoma is an increase in intraocular pressure (IOP). When IOP is elevated, the depression in the optic nerve head $(\mathrm{ONH})$, which is called a cup, is enlarged, and the ratio of the diameters of the cup to the $\mathrm{ONH}$, also called as disc, becomes large. This cup-to-disc (C/D) ratio is, in fact, one of the important signs of glaucoma that can be found on the retinal fundus images. However, the determination of C/D ratio is not always easy because the contrast between cup regions and the surrounding regions (rim) is usually low. The intra- and inter-reader variations in the determination of $\mathrm{C} / \mathrm{D}$ ratios can become large $[7,8]$.

The three dimensional (3D) analysis of $\mathrm{ONH}$ is expected to improve the diagnosis of glaucoma. For development of the CAD system for glaucoma diagnosis, the 3D images of the $\mathrm{ONH}$ were reconstructed by use of stereo images in the previous study $[9,10]$. In this study, the cup and disc regions were determined by use of the depth images, and the C/D ratios were used for distinguishing between glaucomatous and non-glaucomatous eyes.

\section{MATERIALS AND METHODS}

\subsection{Retinal fundus image database}

Images used in this study were taken by a stereo retinal fundus camera (prototype of the WX-1, Kowa Company, Ltd., Tokyo, Japan). A pair of images of an eye was taken consecutively from two different angles. Eighty image pairs including 25 cases with glaucomatous $\mathrm{ONH}$ and 55 cases without the signs of glaucoma were included. The outlines of cup and disc regions were provided manually by an experienced ophthalmologist on a stereo display using 3D glasses, which are considered as the "gold standard" in this study. For the subset of 44 cases including the 24 glaucoma cases, the outlines were provided by another experienced ophthalmologist for evaluation of the intra-reader variation.

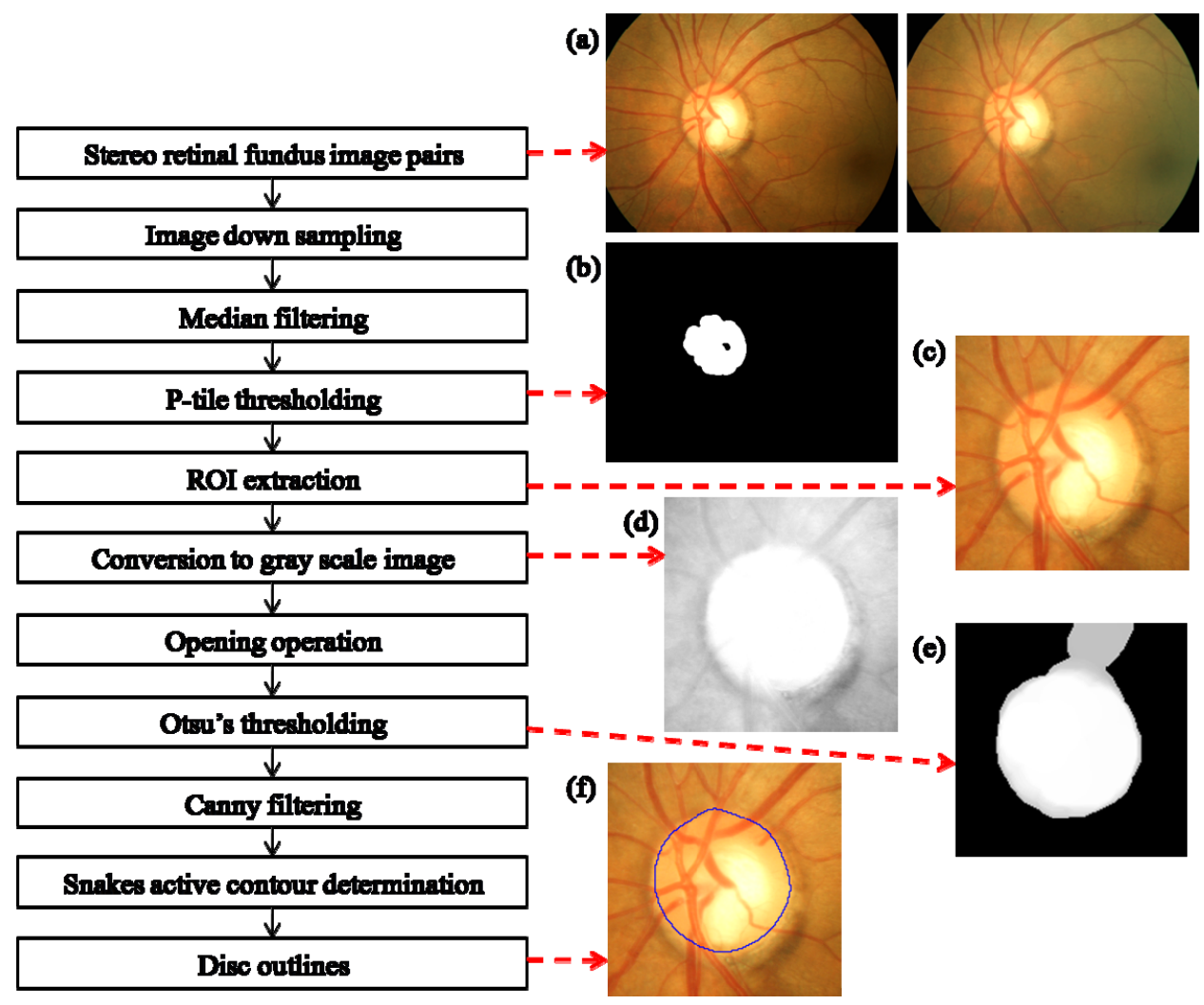

Fig. 1. Flowchart of the procedure for disc region determination and the corresponding processed images: (a) original image pair, (b) p-tile thresholded image, (c) ROI including the ONH, (d) gray-scaled image based on red channel, (e) Otsu's thresholded image, and (f) ROI with the disc outline 


\subsection{Determination of disc outlines}

The method for the determination of disc region is outlined in Fig. 1. First, the region of interest (ROI) including the ONH was extracted from a retinal fundus image for the subsequent processing of the precise disc determination. The ONH regions can be identified with high pixel values in the red color channel of the RGB color images and cover approximately $3.6 \%$ of the retinal fundus images in this study. Therefore, an approximate ONH region was identified by use of the p-tile thresholding method with the red color channels. For reducing the noise and the processing time, the images were down sampled by pixel averaging and median filtered. The largest identified region with the circularity larger than the prespecified value ( 0.45 in this study) in each of a pair of images was determined as the approximate location of the $\mathrm{ONH}$, and their center was employed as the center of the ROI.

The gray-scale images were created based on the red color component. After the opening operation, the Otsu's thresholding method was employed for enhancing the contrast between the ONH regions and the retinal regions. Subsequently, the border candidate pixels were identified using the Canny edge detector. Finally the outlines of the discs were determined by use of the Snakes active contour method followed by the spline operation. Figure 1 also shows an original retinal fundus image pair, p-tile thresholded image, the identified ROI including the ONH region, the grayscale image based on the red color component, the image after the Otsu's thresholding method, and the ROI with the outline of the disc determined by this method.

\subsection{Determination of cup outline}

The cup regions were determined based on the 3D structural information. The method for creating the depth images of ONH regions is explained in detail elsewhere $[9,10]$. Briefly, the corresponding pixel locations in a pair of stereo images were determined based on the cross-correlation in the localized regions. The depth at each pixel is calculated based on the distance (disparity) between these corresponding points by use of the equations

$$
\begin{gathered}
\text { Depth }=\frac{L \times \tan \left(\theta-\beta_{L}\right) \times \tan \left(\theta+\beta_{R}\right)}{\tan \left(\theta-\beta_{L}\right)+\tan \left(\theta+\beta_{R}\right)} \\
\beta_{L}=\tan ^{-1}\left\{x_{L} \times \tan \left(\frac{\alpha}{2} \times \frac{\pi}{180}\right) \times \frac{2}{W}\right\} \\
\beta_{R}=\tan ^{-1}\left\{x_{R} \times \tan \left(\frac{\alpha}{2} \times \frac{\pi}{180}\right) \times \frac{2}{W}\right\}
\end{gathered}
$$

and

$$
x_{R}=x_{L}+\text { disparity, }
$$

where $\mathrm{x}_{\mathrm{L}}$ and $\mathrm{x}_{\mathrm{R}}$ area the horizontal coordinates of the corresponding points in the left and right images, respectively, and $\alpha, \beta, \mathrm{W}$, and $\mathrm{L}$ are the angle of view, the angle between the position of the corresponding point and the optical axis, the width of the images, and the length between the optical centers of the camera, respectively. Because the contrast in red color component inside the ONH is low as shown in Fig. 1(d), the cross-correlation coefficients were determined only by use of the green and blue color pixel values. If the cross-correlation value was too low or the contrast inside the localized region was small, the corresponding location was considered not found, and the disparity was interpolated by the surrounding pixels.

Figure 2 shows the flowchart of the cup determination procedure. Based on the depth calculated as above, the deepest pixel inside the disc region was found and considered as the center of the cup region. The depth image in Cartesian coordinate system was transformed in polar coordinate system with the center as a fulcrum. The strongest edges in the radial direction were considered as the initial candidate points for the cup border. The regions outside the detected disc regions were excluded from the candidates. The final candidate points for the cup outline were determined based on the edge strength and the smoothness of the border. Depth images in the Cartesian coordinate system and the polar coordinate system, the edge enhanced image, the image with the initial candidate points for the cup outline, and the image with the determined cup outline are also shown in Fig. 2. 


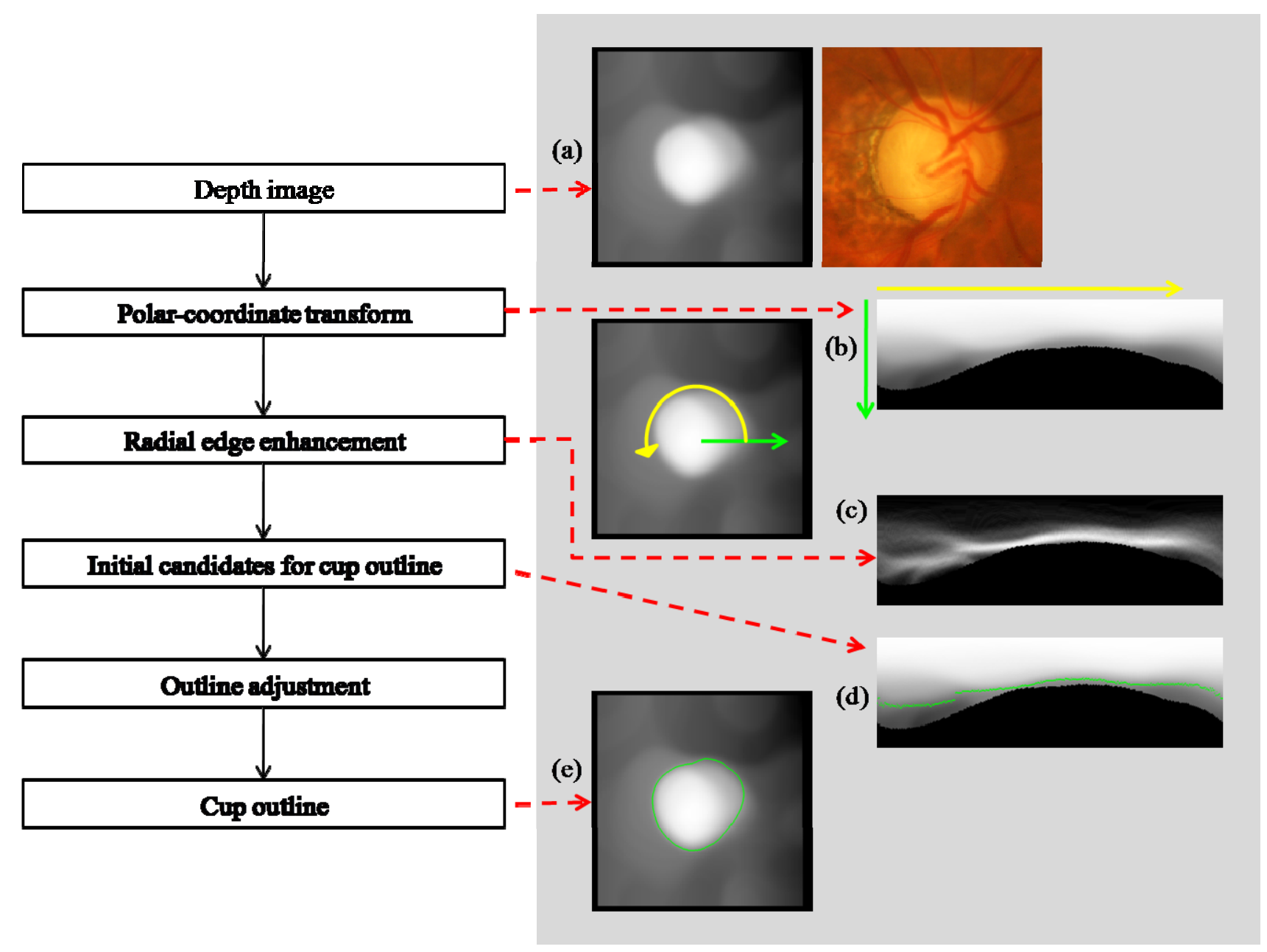

Fig. 2. Flowchart of the cup determination method and the corresponding processed images: (a) depth image along with the original image, (b) depth image in the polar coordinate system, (c) edge enhanced image, (d) image with the initial candidate points for cup border, and (e) depth image with the determined cup outline.

The results for determined cup and disc regions are compared with those based on the ophthalmologist's outlines in terms of the concordance rate, which is defined as the ratio of the area of intersection to the area of union. The C/D ratios were computed by measuring the longest vertical lengths inside the cup and disc regions. The ability for distinguishing between the glaucomatous $\mathrm{ONH}$ and non-glaucomatour $\mathrm{ONH}$ by use of the $\mathrm{C} / \mathrm{D}$ ratios was also evaluated.

\section{RESULTS}

\subsection{Agreement of cup and disc regions between two ophthalmologists}

For the subset of 44 cases, the concordance rate for the disc regions based on the outlines by the two ophthalmologists was high, 0.92. The concordance rates were comparable for the glaucoma and non-glaucoma cases. On the other hand, the concordance rate for the cup regions was somewhat lower, especially for the non-glaucoma cases. The one of the reasons can be that the depth at the cup borders for non-glaucoma cases usually change gradually, and one may consider the beginning of the slope as the cup borders, whereas the other may consider the middle of the slope as the cup borders. In this study, one of them consistently drew the outlines larger. The concordance rates for the cup and disc regions are summarized in Table 1. 
Table 1. The concordance rates for the disc and cup regions determined based on the outlines provided manually by two ophthalmologists. The concordance rate was defined as the ratio of the area of intersection to the area of union.

\begin{tabular}{lcc}
\hline & Disc regions & Cup regions \\
\hline Glaucoma cases & 0.92 & 0.73 \\
\hline Non-glaucoma cases & 0.92 & 0.52 \\
\hline All cases & 0.92 & 0.63 \\
\hline
\end{tabular}

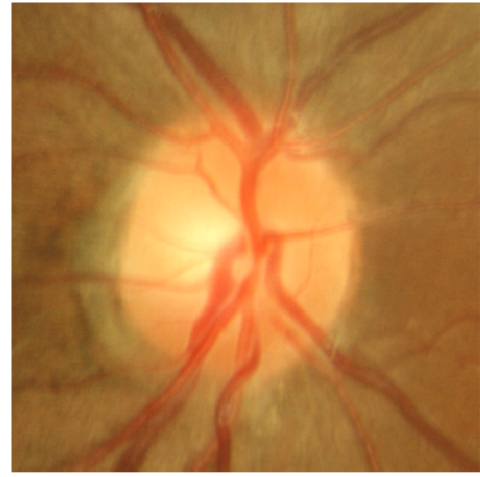

(a)

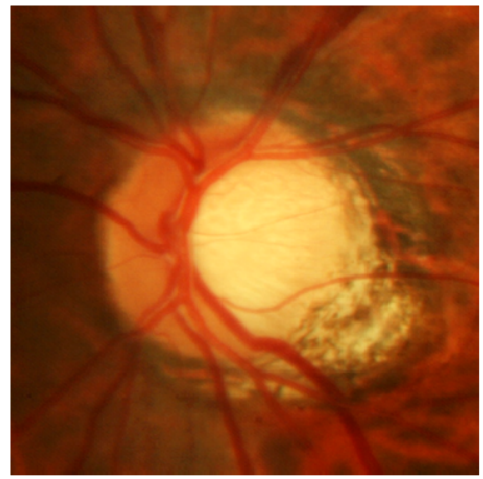

(d)

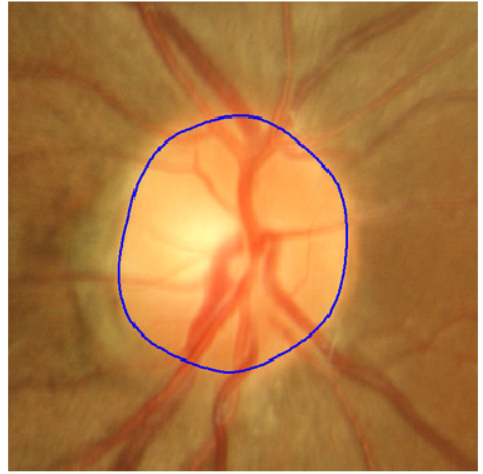

(b)

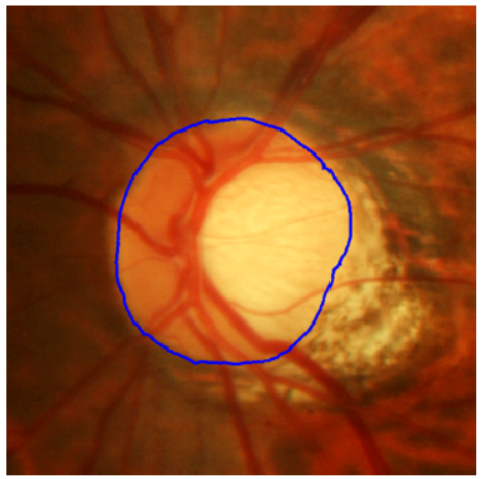

(e)

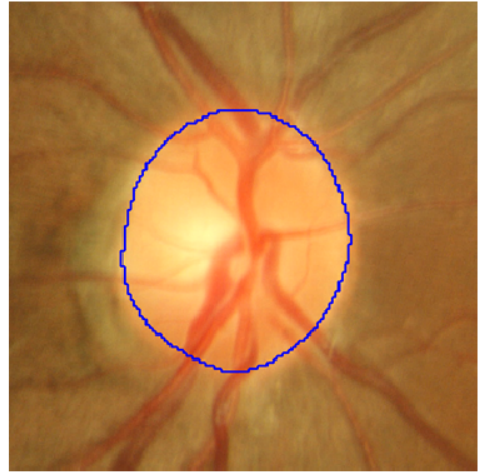

(c)

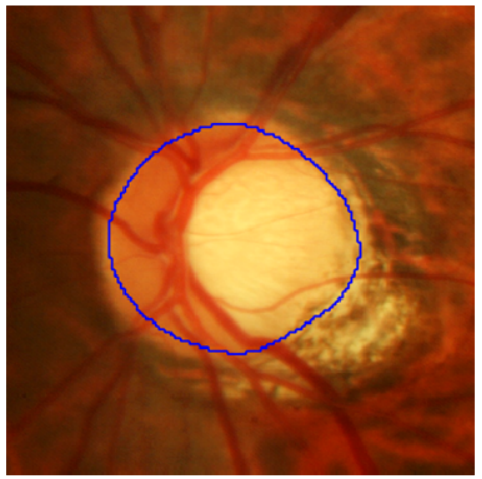

(f)

Fig. 3. (a) Non-glaucoma case (b) with the disc outline by the ophthalmologist and (c) with that by the computerized method. The concordance rate was 0.94. (d) Glaucoma case with the PPA. The disc outlines (e) by the ophthalmologist and (f) by the computerized method. The concordance rate was 0.84 for this case.

\subsection{Evaluation of the cup and disc regions determined by the computerized method}

The average concordance rate for the disc regions determined by the computerized method and those of the gold standard for the 80 cases was 0.83 , which was relatively high. The results were slightly higher for the non-glaucoma cases. The computerized method sometimes over-extracted the areas of peipappillary choriretinal atrophy (PPA), which is more frequently seen in the images of glaucoma patients. Figure 3 shows the non-glaucoma and glaucoma cases with the disc outlines by the ophthalmologist and by the computerized method. It is apparent in Fig. 3(f) that a part of the PPA region was included inside the disc outline determined by this method. Because the PPAs are usually seen in the ear side, i.e., in the horizontal side, of the ONH, over-estimation of the disc regions due to the PPAs is less likely to influence the determination of the $\mathrm{C} / \mathrm{D}$ ratios. 
The determination of cup regions was generally more difficult, as evidenced by the lower concordance rate between the ophthalmologists for the cup regions than for the disc regions. The average concordance rate for the cup regions determined by the computerized method and those of the gold standard was 0.51 , which was slightly lower than that between the ophthalmologists. In some cases, the depth determination results were suboptimal because the corresponding pixels in pairs of images were not found or found incorrectly. In some other cases, the edges of the cups were not identified correctly. The concordance rate for the glaucoma cases was slightly higher than that for the nonglaucoma cases. For the non-glaucoma cases, the cup regions determined by this method tended to be larger than those determined by the ophthalmologist. Figure 4 shows the original ROIs, the depth images, and the images with outlines by the ophthalmologist and by the computerized method. The concordance rates were relatively high for the upper two cases; however, the improvement is needed for the lower two cases. The average concordance rates for the disc and cup regions are summarized in Table 2.

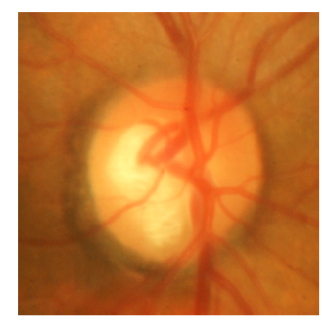

(a)

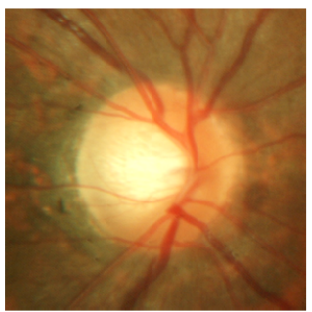

(b)

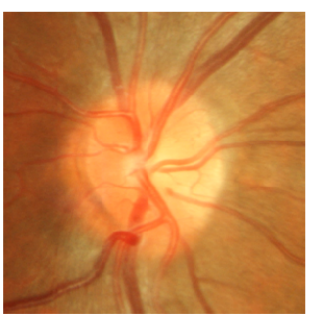

(c)
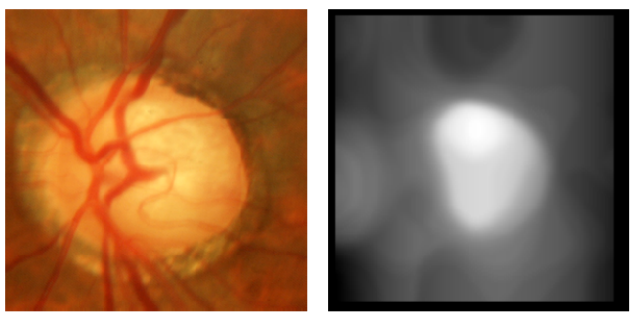

(d)
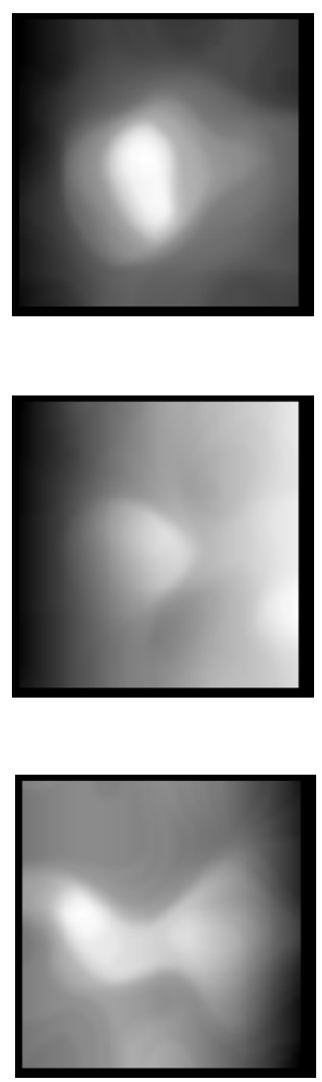
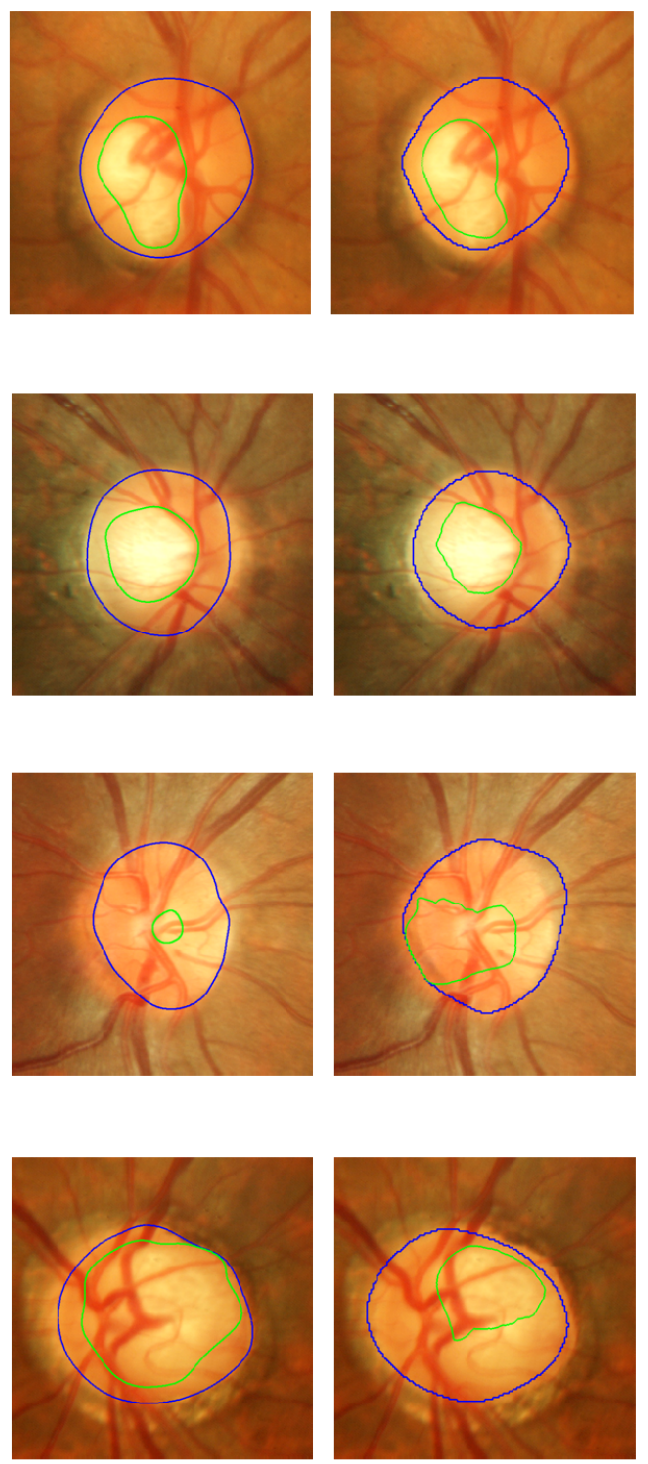

Fig. 4. Glaucoma cases (a), (d) and non-glaucoma cases (b), (c) and their corresponding depth images and the images with the cup and disc outlines by the ophthalmologist and by the computerized method. 
Table 2. The concordance rates for the disc and cup regions determined by the computerized method and based on the outlines by the ophthalmologist. The concordance rate was defined as the ratio of the area of intersection to the area of union.

\begin{tabular}{lcc}
\hline & Disc & Cup \\
\hline Glaucoma cases & 0.82 & 0.60 \\
\hline Non-glaucoma cases & 0.85 & 0.47 \\
\hline All cases & 0.83 & 0.51 \\
\hline
\end{tabular}

\subsection{Classification of glaucoma and non-glaucoma cases based on the $\mathrm{C} / \mathrm{D}$ ratio}

The ability for distinguishing between the glaucoma and non-glaucoma cases was evaluated by use of the receiver operating characteristic (ROC) analysis. When the ratios of the largest vertical diameters of cup and disc regions were employed, the area under the ROC curve was 0.83 , which is relatively high. By use of this method, the $88 \%$ of the glaucoma cases can be correctly identified as glaucoma, whereas $65 \%$ of the non-glaucoma cases can be correctly classified as non-glaucoma.

\section{DISCUSSION}

The disc regions in the retinal fundus images can be determined relatively well by use of the red color channel of the RGB color images. The determination of the cup regions is more difficult; the variation between the experienced ophthalmologists was also large, because there was no solid guideline. However, there is a potential for quantifying the difference in cup shapes between the glaucoma and non-glaucoma cases by use of the depth images.

Other groups have also investigated the methods to analyze the ONH by use of stereo fundus images. Abramoff et al. [11], instead of determining the borders of cup and disc, attempted to classify each pixel as either rim, cup, or background. The accuracy of the pixel classification was $88 \%$, and the correlation between the C/D ratios determined based on the reference standard and the computerized method was 0.93 . The study by Xu et al. [12] was similar to ours. They reconstructed the 3D images and determined the cup borders by the thresholding method. By use of the vertical $\mathrm{C} / \mathrm{D}$ ratio, the AUC for distinction between the glaucomatous and healthy eyes was 0.81 on 54 cases.

In this study, we attempted to determine the cup outlines by finding the strong edges in the depth structure. By use of this method, AUC of 0.83 for distinguishing between glaucomatous and non-glaucomatous eyes were obtained. The disc and cup determination method should be further improved and tested on the larger database. The computerized determination of the $\mathrm{C} / \mathrm{D}$ ratios can be useful for diagnosis of glaucoma on the stereo retinal fundus images.

\section{ACKNOWLEDGMENT}

The authors are grateful to K. Ishida, M.D. for providing the manual outlines of the cups and discs for a subset of cases. This study was supported in part by a grant for the Knowledge Cluster Creation Project from the Ministry of Education, Culture, Sports, Science and Technology, Japan under the heading "Gifu-Ogaki Area: Robotics Pioneering Medical Care Cluster". 


\section{REFERENCES}

[1] Fujita, H., Uchiyama, Y. Nakagawa, T., Fukuoka, D., Hatanaka, Y., Hara, T., Lee, G. N., Hayashi, Y., Ikedo, Y., Gao, X., and Zhou, X., "Computer-aided diagnosis: The emerging of three CAD systems induced by Japanese health care needs," Comput. Methods Programs Biomed. 92, 238-248 (2008).

[2] Hayashi, Y., Nakagawa, Y., Hatanaka, Y., Aoyama, A., Kakogawa, M., Hara, T., Fujita, H., and Yamamoto, T., "Detection of retinal nerve fiber layer defects in retinal fundus images using Gabor filtering," Proc. SPIE 6514, 65142Z1-8 (2007).

[3] Hatanaka, Y., Nakagawa, T., Hayashi, Y., Mizukusa, Y., Fujita, A., Kakogawa, M., Kawase, K., Hara, T., and Fujita, H., "CAD scheme to detect hemorrhages and exudates in ocular fundus images," Proc. SPIE 6514, 65142M-1-8 (2007).

[4] Resnikoff, S., Pascolini, D., Etya'ale, D., Kocur, I., Pararajasegaram, R., Pokharel, G. P., and Mariotti, S. P., "Global data on visual impairment in the year 2002," Bulletin of the WHO 82, 844-851 (2004).

[5] Quigley, H. A. and Broman, A. T., "The number of people with glaucoma worldwide in 2010 and 2020 ," Br. J. Ophthalmol. 90, 262-267 (2006).

[6] Hattenhauer, M. G., Johnson, D. H., Ing, H. H., Herman, D. C., Hodge, D. O., Yawn, B. P., Butterfield, L. C., and Gray, D. T., "The probability of blindness from open-angle glaucoma," Ophthalmology 105, 2099-2104 (1998).

[7] Varma, R., Spaeth, G. L., Steinmann, W. C., and Katz, L. J., “Agreement between clinicians and an image analyzer in estimating cup-to-disc ratios," Arch Ophthlmol. 107, 526-529 (1989).

[8] Tielsch, J. M., Katz, J., Quigley, H. A., Miller, N. R., and Sommer, A., "Intraobserver and interobserver agreement in measurement of optic disc characteristics," Ophthalmology 95, 350-356 (1988).

[9] Nakagawa, T., Hayashi, Y., Hatanaka, Y., Aoyama, A., Hara, T., Kakogawa, M., Fujita, H., and Yamamoto, T., "Comparison of the depth of an optic nerve head obtained using stereo retinal images and HRT," Proc. SPIE 6511, 65112M1-M9 (2007).

[10] Nakagawa, T., Suzuki, T., Hayashi, Y., Mizukusa, Y., Hatanaka, Y., Ishida, K., Hara, T., Fujita, H., and Yamamoto, T., "Quantitative depth analysis of optic nerve head using stereo retinal fundus image pair," J. Biomed. Optics 13, 064026-1-10 (2008).

[11] Abramoff, M. D., Alward, W. L. M., Greenlee, E., C., Shuba, L., Kim, C. Y., Fingert, J. H., and Kwon, Y. H., "Automated segmentation of the optic disc from stereo color photographs using physiologically plausible features," Invest. Ophthalmol. Vis. Sci., 48, 1665-1673 (2007).

[12] Xu, J., Ishikawa, H., Wollstein, G., Bilonick, R. A., Sung, K. R., Kagemann, L., Townsend, K. A., Schuman, J. S., "Automated assessment of the optic nerve head on stereo disc photographs," Invest. Ophthalmol. Vis. Sci., 49, 2512-2517 (2008). 